\title{
GaN HEMT Class-E Rectifier for DC+AC Power Recovery
}

\author{
M. Nieves Ruiz *, David Vegas *, José R. Pérez-Cisneros ${ }^{* \dagger}$, José A. García * \\ ${ }^{*}$ Department of Communications Engineering, University of Cantabria, 39005 Santander, Spain \\ ${ }^{\dagger}$ Aragon Institute of Engineering Research (I3A), University of Zaragoza, 50018 Zaragoza, Spain
}

\begin{abstract}
A 915 MHz GaN HEMT-based class-E rectifier is proposed in this paper to be used for $\mathrm{DC}+\mathrm{AC}$ wireless power recovery. Taking advantage of the time reversal (TR) duality principle, the rectifier was derived from a class-E inverter, whose output network was designed for high-efficiency operation over a wide range of resistive loads. The addition of an appropriate gateside termination allows the device to be turned-on without an additional RF source for gate driving. The rectifier reduced sensitivity to load variation, as well as its capability for efficiently and linearly recovering the envelope of an AM RF excitation, were then characterized. An average efficiency of $82 \%$ has been measured for the combined RF-to-DC and RF-to-AC power conversion of a $1.6 \mathrm{~W}$ modulated carrier. Frequency multiplexing and frequency modulation alternatives for high-level $\mathrm{DC}+\mathrm{AC}$ wireless power transmission are finally presented.
\end{abstract}

Index Terms - Class-E, GaN HEMT, rectifier, wireless power transmission.

\section{INTRODUCTION}

Far-field wireless power transmission (WPT) links are conceived for the remote recovery of DC power, mainly motivated by the increased interest on powering wireless sensors, RFID tags, or many other DC loads requiring low voltage and current levels. Although the grid and most common power-demanding loads operate with $\mathrm{AC}$, the case for instance of induction motors, implementing linear and efficient AC-to-RF and RF-to$\mathrm{AC}$ power converters still remains as a challenging task [1]. Thus, intermediate conversions from AC-to-DC and from DCto-AC, with more than mature implementations and good performance, are common at both sides of the link.

In [2], the integration of two class-E power amplifiers and a pair of identical class-E rectifiers, making use of center-tap transformers, allowed the simultaneous transmission of both semi-sinusoids in the utility waveform, based on well-known $\mathrm{AM}$ and polarization multiplexing concepts. However, the use of diode-based rectifiers in the receiving side imposes a relevant limitation for high power recovery. If considering that fast switching Schottky diodes with high voltage and current capability are rarely available at the $915 \mathrm{MHz}, 2.45 \mathrm{GHz}$ or $5.8 \mathrm{GHz}$ ISM frequency bands, those rectifiers could only deliver low levels to the DC and AC loads (a few tens of milliwatts according to the device and levels reported in [2]).

A GaN HEMT-based rectifier is proposed in this paper, as the choice for high power recovery. Considering the extreme sensitivity of class-E topologies to load variations [3] and that the transistor could be even destroyed under extreme loading conditions (not so uncommon in applications like fluorescent lamps, electric heating, etc.), a load-invariant topology has been selected. Additionally, a self-synchronous operation [4] has been forced to avoid the need for an auxiliary RF source to drive its gate terminal, not usually available at the remote position. Besides validating its capability for DC and AC power recovery at a few watts, alternatives to the $\mathrm{AM} /$ polarization multiplexing technique in [2] are also tested.

\section{SELF-SYNCHRONOUS RECTIFIER}

Taking advantage of the time reversal duality principle [5], a rectifier with reduced sensitivity to load variation was derived from a class-E power amplifier (PA), designed for maximum efficiency under load modulating conditions. Considering the available nonlinear models for $\mathrm{RF} /$ microwave FET devices may fail when reproducing the third quadrant of their voltamperic characteristics, this could be the only way to proceed unless an own dedicated model were extracted.

\section{A. Drain Terminating Network and PA Design}

A CGH35030F GaN HEMT packaged device from Wolfspeed was selected for the designs. After adjusting the input power level to force a switch-type of operation, loadpull simulations at the fundamental, $915 \mathrm{MHz}$, were completed (see Fig. 1). A nearly infinite choke was used, with open circuit terminations forced at the higher order harmonics. As it could be expected from [6], a trajectory with maximum efficiency and variable output power exists. Under the theoretical assumptions $R_{O N}=0$ and $R_{O F F}=\infty$, this impedance path would result in zero voltage but variable slope voltage switching at turn on.

A drain terminating network was then designed, able to approximate the optimum path under varying load condition, $R_{L}$. A modification to the inductive impedance inverter in [3] was employed, as presented in the inset of Fig. 1.

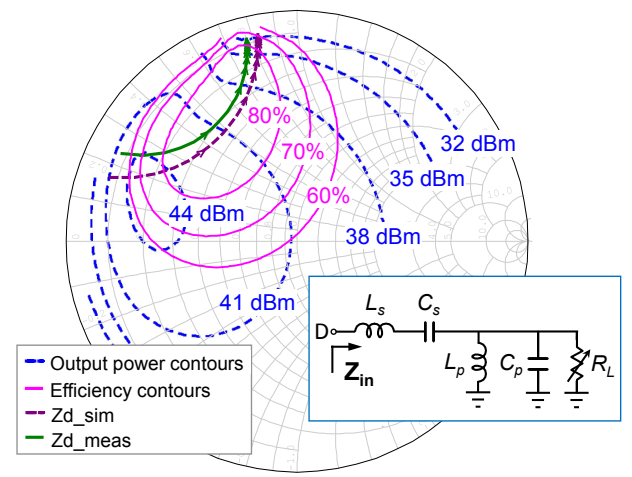

Fig. 1. Efficiency (-) and output power (--) load-pull contours from simulations, together with the simulated (-) and measured (-) $Z_{\text {in }}(f)$ evolution with $R_{L}$. The drain-side network schematic has been included in the inset. 
The evolution with $R_{L}$ of its input impedance, simulated and measured for a 5-500 $\Omega$ range, is also plotted over the contours in Fig.1. The lumped-element parasitics have been taken into consideration.

The class-E PA design was completed with the input matching and biasing networks. A peak efficiency of $83.2 \%$ was obtained at $75 \Omega$, staying above $70 \%$ along a resistive termination range from 20 to $200 \Omega$ (over $8 \mathrm{~dB}$ of power backoff).

\section{B. Self-synchronous Rectifier Derivation}

The circuit schematic for the designed class-E topology, once prepared for its use as rectifier, is shown in Fig. 2. In order to guarantee a self-synchronous operation, the gate-side circuit was slightly modified to synthesize the required inductive termination, calculated according to [7]. Coils and capacitors are high Q parts from Coilcraft and ATC, respectively.

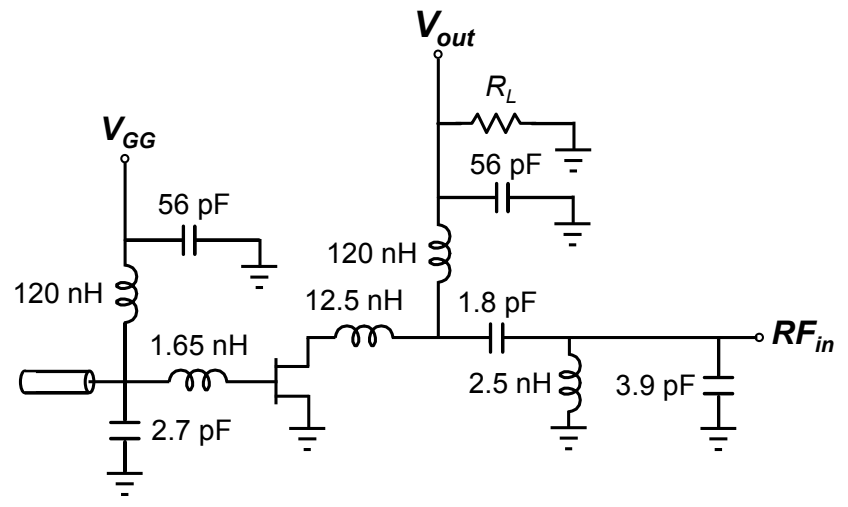

Fig. 2. Schematic and component values for the designed rectifier.

\section{Continuous Wave (CW) Characterization}

Once the self-synchronous operation was accomplished, the rectifier was characterized with a $915 \mathrm{MHz} \mathrm{CW}$ excitation. A peak overall efficiency of $84.4 \%$ was measured for an $85 \Omega$ load resistance, corresponding to a $21.5 \mathrm{~V}$ DC output voltage. As it can be appreciated from Fig. 3a, the recovered voltage linearly follows the amplitude of the RF input signal, with an efficiency value better than $80 \%$ for input power levels above $32 \mathrm{dBm}$ $($ Vin $>24 \mathrm{~V})$. This particular feature makes it attractive for being used as high-level envelope detector.

The sensitivity of the rectifier to a varying load may be observed from the results in Fig. 3b. Although a significant variation of $V_{\text {out }}$ is appreciated, the peak in the drain-to-source voltage waveform $\left(V_{d s \text { peak }}=3.562 \cdot V_{\text {out }}\right.$ for nominal operation $)$ is kept below the device breakdown voltage (better than $120 \mathrm{~V}$ ).

The degradation in efficiency, when compared to its use as PA in section IIA, is due to the input mismatch. A class-E rectifier of this kind, designed for a load-independent high-efficiency performance, is expected to offer a resistive input impedance. Although not included in this work, the mismatching losses may be reduced with the use of a resistive compression network and a plurality of similar rectifiers [8]. a)

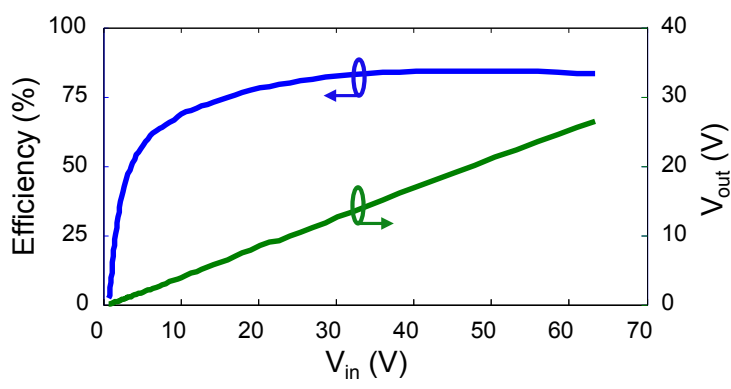

b)

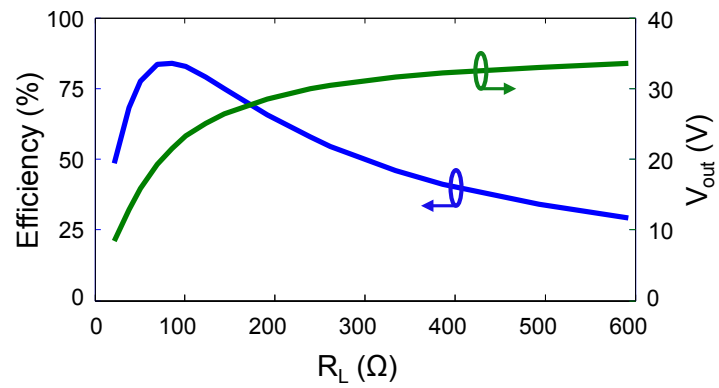

Fig. 3. Measured profiles for the efficiency and the rectified voltage vs the amplitude of the $915 \mathrm{MHz}$ excitation a) and the DC load resistance b).

\section{RF-TO-DC+AC POWER CONVERSION}

In order to exploit the capability of the selected topology for linearly and efficiently working as envelope demodulator (Fig. 3a), two similar self-synchronous rectifiers were combined to recover a $50 \mathrm{~Hz}$ waveform, based on the low power approach in [2]. The implemented measurement setup is shown in Fig. 4, where two $915 \mathrm{MHz}$ carriers, amplitude modulated with each of the semi-sinusoids, were conveniently amplified and employed as excitations. The high-level demodulated waveforms were captured with an oscilloscope, while the total RF input power was measured with the aid of two power sensors.

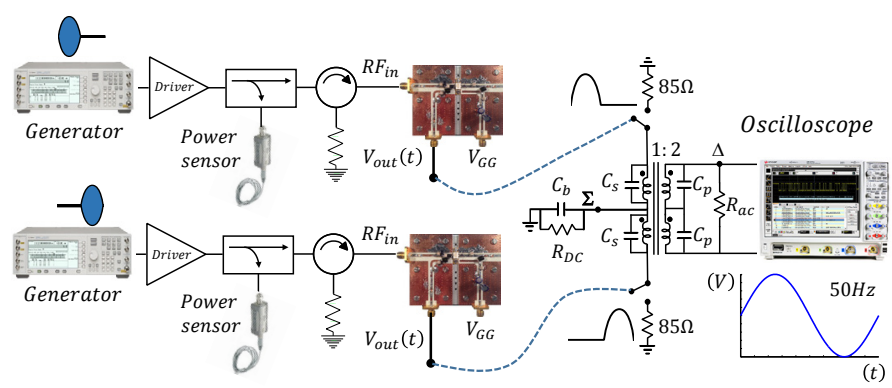

Fig. 4. Measurement set-up for characterizing the RF-to-DC+AC power converter, resulting from combining two self-synchronous rectifiers.

The recovered semi-sinusoids were first captured with the rectifiers loaded by the optimum $R_{L}=85 \Omega$ value from Fig. 3 b. Shown in Fig. 5, no significant distortion is appreciated with peak values around $21 \mathrm{~V}$ for an average input power of $32 \mathrm{dBm}$ in each branch (peak levels $6 \mathrm{~dB}$ higher). Under this loading condition, the average output power per branch was as high as $1.32 \mathrm{~W}$, for a global efficiency around $82 \%$. 
With the aim of extracting the $\mathrm{DC}$ and $\mathrm{AC}$ components through mutually isolated output ports, a COTS center-tap transformer with a 2:1 ratio between the primary and secondary windings was added. Its connection in Fig 4 allows stepping-up the voltage. The winding magnetization inductances were measured at $50 \mathrm{~Hz}$ for appropriately selecting the required $C_{s}$ and $C_{p}$ resonating capacitors. A high-valued capacitor of 10000 $\mu \mathrm{F}$ was employed for bypassing the DC load $\left(C_{b}\right)$. The waveform at the AC port, captured with the oscilloscope, has been added to Fig. 5. Although not represented, a DC voltage value of $6.7 \mathrm{~V}$ was measured at the $\Sigma$ (DC) output port.

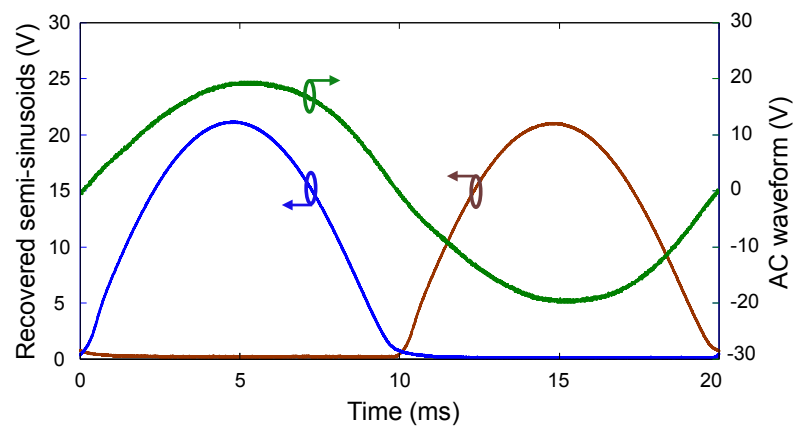

Fig. 5. Demodulated semi-sinusoids under optimum resistive loading. The measured waveform at the $\Delta$ port, when adding the transformer, is included.

\section{DC+AC WPT ALTERNATIVES}

As alternatives to the use of AM modulation together with polarization multiplexing in [2], two classic analog communication techniques were also tested for the receiving part of a WPT link, using the above described RF-to-DC+AC power converter: amplitude modulation combined with frequency multiplexing, and frequency modulation. Fig. 6 shows a photograph of the implemented measurement setup, common to both approaches. A $40 \mathrm{~W}$ cavity duplexer for $900 \mathrm{MHz}$ E-GSM band operation constitutes the core element added to the diagram in Fig. 4.

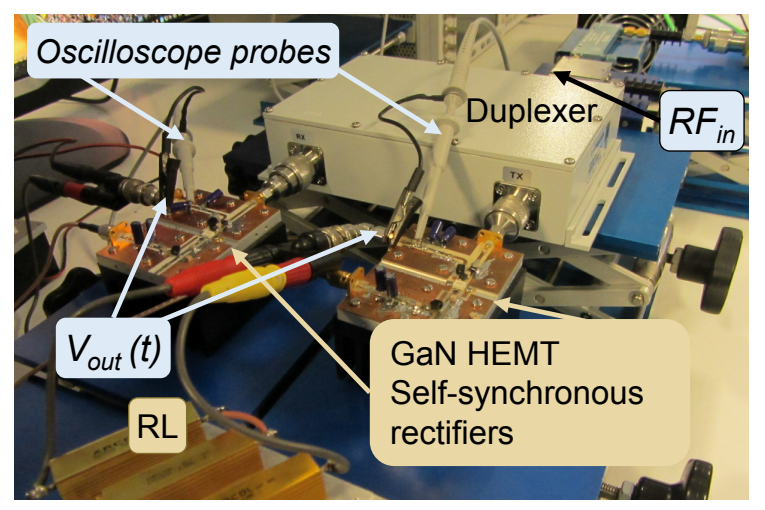

Fig. 6. Photograph of the implement set-up, including details of the GaN HEMT rectifiers and the cavity duplexer.

\section{A. AM with Frequency Multiplexing}

Considering the passbands of a GSM duplexer (880 - 915 $\mathrm{MHz}$ and $925-960 \mathrm{MHz}$ ), as well as the $26 \mathrm{MHz}$ available bandwidth for the $915 \mathrm{MHz}$ ISM band, two carriers at 902 and 928 $\mathrm{MHz}$ may be amplitude modulated with their respective semisinusoids. Notice that the bandwidth of an AM signal, 100\% modulated by a semi-cycle of the $50 \mathrm{~Hz}$ waveform, is limited to a few hundreds of Hz. The generated AM RF components were combined and amplified to reproduce the conditions to be obtained at the antenna port in the receiving side of the link. A total average power of $41 \mathrm{dBm}$ was applied to the duplexer input $\left(R F_{\text {in }}\right.$ port detail in Fig. 6). The modulating semi-sinusoids were recovered without appreciable distortion by the rectifier circuits, with $72.2 \%$ and $68.5 \%$ of average efficiency values. The measured efficiency reduction, if compared to section III results, is due to duplexer insertion losses.

\section{B. FM approach}

The AC waveform may be also demodulated from a constant envelope FM signal. If using a carrier at $919.5 \mathrm{MHz}$ with 8.5 $\mathrm{MHz}$ of frequency deviation, the same cavity duplexer may be employed as a frequency discriminator. Following an old and proven idea [9], the utility waveform or any other modulating signal could be extracted, with a trade-off between linearity and efficiency. With $6.3 \mathrm{~W}$ of RF input power, 1.9 and $2 \mathrm{~W}$ of average output power were obtained at the outputs of the rectifiers, resulting in this case in a total efficiency for the $\mathrm{DC}+\mathrm{AC}$ recovered power about $62 \%$.

\section{CONCLUSION}

A GaN HEMT-based Class-E rectifier has been proposed for its use in high-level $\mathrm{DC}+\mathrm{AC}$ wireless power recovery. The drain-side network has been designed for an efficient operation over a wide range of resistive loads, while an inductive gate side termination allows the desired self-synchronous switching. Its reduced sensitivity to load variation has been shown, as well as its capability for efficiently and linearly recovering the envelope of an AM RF excitation with an average efficiency of $82 \%$. Frequency multiplexing the AM components or frequency modulating with the utility waveform, alternative high-level DC+AC WPT links may be configured. The proposed architectures, restricted to the receiving side, could be also employed for recovering a higher frequency waveform, the case of the hundreds of $\mathrm{kHz}$ or few $\mathrm{MHz}$ switching waveforms of inductive and resonant WPT systems.

\section{ACKNOWLEDGMENT}

This work was supported by the Ministry of Economy and Competitiveness (MINECO) through TEC2014-58341-C4-1-R and TEC2014-58341-C4-2-R projects, co-funded with FEDER. The authors wish to thank Prof. Christian Brañas, University of Cantabria, by his assistance with the transformer characterization. David Vegas and José R. Pérez-Cisneros also thank the 
support provided by the pre-doctoral BES-2015-072203 and the pre-doctoral mobility EEBB-I-15-10447 grants, respectively.

\section{REFERENCES}

[1] N. Shinohara, Wireless Power Transfer via Radiowaves, London: J. Wiley \& Sons, 2014.

[2] J.R. Pérez-Cisneros, M.N. Ruiz, M. Lobeira, C. Brañas, J. de Mingo, J.A. García "Class-E Power Converters for AC $(50 / 60 \mathrm{~Hz})$ Wireless Transmission," IEEE MTT-S International Microwave Symp., San Francisco, May 2016.

[3] M. K. Kazimierczuk and Xung T. Bui, "Class E Amplifier with an Inductive Impedance Inverter," IEEE Trans. Industrial Elect., vol. 37, no. 2, pp. 160-166, April 1990.

[4] M. Roberg, T. Reveyrand, I. Ramos, E. A. Falkenstein and Z. Popovic, "High Efficiency Harmonically Terminated Diode and Transistor Rectifiers," IEEE Trans. Microwave Theory Tech., vol. 60, pp. 4043-4052, Dec. 2012.
[5] D. C. Hamill, "Time Reversal Duality and the Synthesis of a Double Class E DC-DC Converter," 21 st Power Electron. Specialist Conf., PESC'90, pp. 512-521, 1990.

[6] M. Acar, A. J. Annema and B. Nauta, "Analytical Design Equations for Class-E Power Amplifiers," IEEE Trans. Circuits Syst. I: Regular Papers, vol. 54, pp. 2706-2717, Dec. 2007.

[7] I. Ramos, M.N. Ruiz, J.A. García, D. Maksimovic, Z. Popovic, "GaN Microwave DC-DC Converters," IEEE Trans. Microwave Theory and Techniques, vol. 63, no.12, pp. 4473-4482, Dec. 2015.

[8] T. W. Barton, J. M. Gordonson and D. J. Perreault, "Transmission Line Resistance Compression Networks and Applications to Wireless Power Transfer," IEEE Journal Emerging Selected Topics Power Elect., vol. 3, pp. 252-260, March 2015.

[9] C. Travis, "Automatic Frequency Control," Proc. IRE, vol. 23, pp. 11251141, Oct. 1935. 